\title{
Rare Infantile Inguinal Hernia Containing Uterus, Ovaries and Fallopian Tubes-A Case Report: Presentation from Yemen
}

\author{
Al-Bahloly M. Abdulhadi' ${ }^{1}$ Al-Bahlooli Saeed Hadi², Mehras Obad Amattalkhaleq33 \\ Kamal Bahakim² \\ ${ }^{1}$ Pediatric Department, Faculty of Medicine, Thamar University, Dhamar, Yemen \\ ${ }^{2}$ Surgical Department, Faculty of Medicine, Thamar University, Dhamar, Yemen \\ ${ }^{3}$ Gynecological Department, Faculty of Medicine, Thamar University, Dhamar, Yemen \\ Email: drsaeedhadi@gmail.com
}

How to cite this paper: Abdulhadi, A.-B.M., Hadi, A.-B.S., Amattalkhaleq, M.O. and Bahakim, K. (2019) Rare Infantile Inguinal Hernia Containing Uterus, Ovaries and Fallopian Tubes-A Case Report: Presentation from Yemen. Surgical Science, 10, 169-172.

https://doi.org/10.4236/ss.2019.105021

Received: April 13, 2019

Accepted: May 26, 2019

Published: May 29, 2019

Copyright $\odot 2019$ by author(s) and Scientific Research Publishing Inc. This work is licensed under the Creative Commons Attribution International License (CC BY 4.0).

http://creativecommons.org/licenses/by/4.0/

\begin{abstract}
The presence of ovaries and fallopian tubes in the sac of female inguinal hernia is rare especially in childhood. However, complete herniation of the uterus side by side with ovaries and tubes constitutes extreme rarity. We herein present a 6-years old female patient whose inguinal hernia contains uterus in addition to ovaries and fallopian tubes. The aim of this report is to attract attention of surgeons to this rare possibility during surgical management of hernia in order to avoid the likely damage to herniated structures.
\end{abstract}

\section{Keywords}

Female Infants, Inguinal Hernia, Uterus, Ovaries

\section{Introduction}

An inguinal hernia in females is relatively uncommon as compared to males. The incidence in females is about $1.9 \%$; the ratio of boys to girls is $6: 1$ [1]. Regarding the content of the hernia sac, about $15 \%-20 \%$ of inguinal hernias in infant girls contain ovary and Fallopian tube; however, the presence of uterus within a hernia sac side by side with the ovaries and fallopian tubes is an extremely rare [2] [3]. Fewer than 10 cases were reported in the literature [2] [4].

To our knowledge, there is nothing known about risk factors for such conditions in the literature. The independent risk factors are positive family history and obstination [3].

Since only few such cases are reported in the literature particularly uterus herniation, we hereby report a case of infant female patient with left inguinal 
hernia that contains even uterus in addition to ovaries and fallopian tubes.

\section{A Case Report}

A 6 years old female infant presented to surgical clinic in Saudi hospital at Hajjah governorate in Yemen in 2013. The chief complaint was a painless swelling in left groin region since birth. The swelling increases in size with crying, straining or longstanding and incompletely disappears spontaneously in lying down or sometimes by gentle compression by the hands. No history of pain, vomiting, abdominal distention or vaginal bleeding. The patient was born normal delivery and full term. By examination in standing position, about $4 \times 4 \mathrm{~cm}$ mass is noted in left inguinal region. In supine position, the size of swelling markedly decreased but remained partially irreducible, so we had to apply gentle compression to reduce the content of the sac back to peritoneal cavity. The swelling was soft, tenderless and the reduction was almost complete. The cough expansile impulse was positive. The finding over the abdomen was normal, so the provisional diagnosis was made as sliding inguinal hernia with bowel content (sigmoid colon). Unfortunately, we regarded ultrasound of the swelling as optional and was not done. The other routine preoperative investigations were normal. The case was registered in the waiting list as elective case and was operated after one month later. Intraoperative finding was surprising for us when we routinely opened the sac. All uterus, tubes and ovaries were freely situated within the hernial sac (Figure 1 and Figure 2). They were fixed by fine adhesions to the inner surface of the sac with no signs of strangulation or damage. The sliding structures were freed up from adhesions and easily reduced back to peritoneal cavity. The sac was highly dissected, transfixed as usual way. The patient was discharged on the $3^{\text {rd }}$ postoperative day and had uneventful recovery.

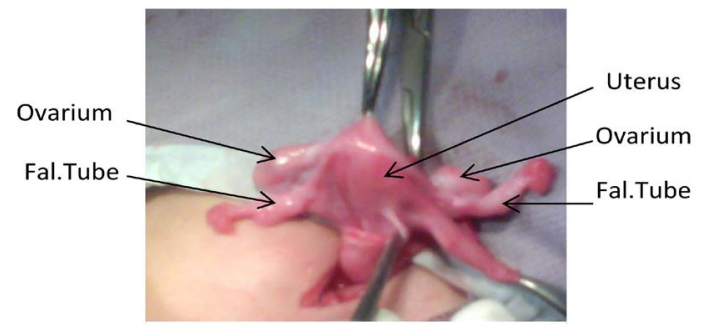

Figure 1. Uterus, fallopian tubes and ovaries found in sac of left inguinal hernia.

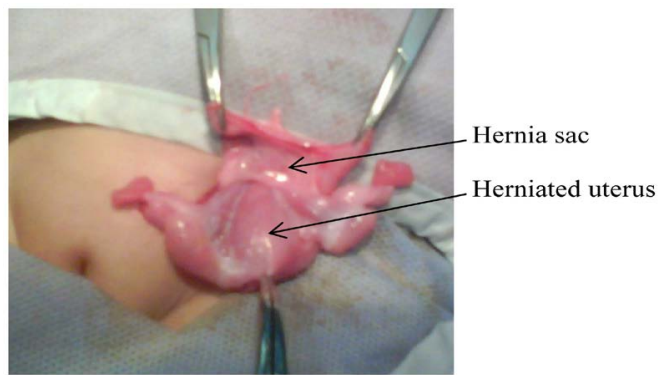

Figure 2. Uterus, fallopian tubes and ovaries found in sac of left inguinal hernia. 


\section{Discussion}

It is known that the inguinal hernia is rare in females compared to males. The incidence of inguinal hernia in females is about $1.9 \%$; the ratio of boys to girls is 6:1 [1]. About 15\% - 20\% inguinal hernias in infant girls contain ovary and fallopian tube [2]. However the presence of uterus within the hernia sac is extremely rare condition in infants [2] [3]. The presentation of an asymptomatic palpable movable mass over the labium major always suggests sliding hernia with ovary. Only few reports have appeared in the literature about hernia sac that contains uterus, fallopian tube, and ovary in female patient [2] [4]. Many of inguinal hernias in female are in fact sliding containing genital structures such as ovaries, fallopian tubes or even the uterus [5] [6]. The hernia sac is usually formed by the unobliterated portion of the prenatal peritoneal invagination of the canal of Nuck that runs along and partly covers the round ligament [7] [8]. Depending on the gender, the processus vaginalis is accompanied by testis or round ligament of the uterus and passes through the inguinal canal toward scrotum or labium major. The processus vaginalis usually disappears by 8 months of gestation [9]. Failure of obliteration of processus vaginalis in female keeps this processus patent and termed as canal of Nuck, which is the cause of hernia [10].

However; the etiology of this pathology is still controversial and there is no clear explanation why the uterus should herniate in girls, unless there is ananatomic abnormality of the ligaments that suspend the uterus [6] [7]. Thomson [11] offered the hypothesis that if there is failure of fusion of the Mullerian ducts that leads to excessive mobility of the ovaries plus nonfusion of the uterine cornuae, the chance of herniation of ovary into the inguinal canal is increased The report added that not only the ovarian herniation can occur but also cases have been documented for herniation of fallopian tube and even herniation of the entire uterus into the inguinal canal of infant females [11].

Because of the risk of damaging herniated structures during the surgical procedure, careful past and family history should be taken as well as performing preoperative investigations. Vaginal bleeding in a child with inguinal hernia may occur when the uterus is the sliding component of the hernia [12]. Therefore, Ming Y C et al. [7], advises performing routinely preoperative ultrasound for all female infants with an irreducible palpable inguinal mass [7]. In our case, neither past history nor family history was relevant. Unfortunately, preoperative ultrasound for our case had not performed since the diagnosis of inguinal hernia was clear. In fact, it was first time in our experience to have such hernia containing internal genital structures. Therefore, we strongly agree with other reports that recommend preoperative ultrasound for any sliding and irreducible hernia. This is because of likely damage to the prolapsed structures.

In conclusion, the presence of genital structures such as ovaries in sliding or irreducible inguinal hernia in female patients is not uncommon, but the presence of uterus and fallopian tubes besides the ovaries in hernia sac is very rare. 
This possibility should be kept in mind of surgeons during physical examination and surgical management of sliding or irreducible inguinal hernia in female. Preoperative ultrasound is recommended for all female with irreducible inguinal hernias to have clear idea about the content of hernia sac, especially when the patient is female infant. Once the diagnosis is conformed, an immediate surgery is required to avoid the unexpected complications.

\section{Conflicts of Interest}

The authors declare no conflicts of interest regarding the publication of this paper.

\section{References}

[1] Read, R.C. and White, J.J. (1978) Inguinal Herniation 1777-1977. American Journal of Surgery, 136, 651-657. https://doi.org/10.1016/0002-9610(78)90329-X

[2] Cascini, V., Lisi, G., Di Renzo, D., et al. (2013) Irreducible Indirect Inguinal Hernia Containing Uterus and Bilateral Adnexa in a Premature Female Infant: Report of an Exceptional Case and Review of the Literature. Journal of Pediatric Surgery, 48, e17-e19. https://doi.org/10.1016/j.jpedsurg.2012.09.065

[3] Daib, A., Boughdir, M., Ben Abdallah, R., Hellal, Y., Ben Malek, M.R., Gharbi, Y. and Kaabar, N. (2017) A Rare Case of Inguinal Utero-Ovarian Hernia in Girls. Journal of Clinical and Medical Case Studies, 2, 1-3.

[4] Huang, C.S., et al. (2003) The Presentation of Asymptomatic Palpable Movable Mass in Female Inguinal Hernia. European Journal of Pediatrics, 162, 493-495. https://doi.org/10.1007/s00431-003-1226-7

[5] Chawla, S. (2001) Inguinal Hernia in Females. Medical Journal Armed Forces India, 57, 306-308. https://doi.org/10.1016/S0377-1237(01)80009-5

[6] Kivilcim, K.C., Rabia, E., Emel, C. and Tolga, E.D. (2015) Inguinal Hernia Containing Uterus, Fallopian Tube, and Ovary in a Premature Newborn: A Case Report. Case Reports in Pediatrics, 2015, Article ID: 807309. https://doi.org/10.1155/2015/807309

[7] Ming, Y.C., Luo, C.C., Chao, H.C. and Chu, S.M. (2011) Inguinal Hernia Containing Uterus and Uterine Adnexa in Female Infants: Report of Two Cases. Pediatrics \& Neonatology, 52, 103-105. https://doi.org/10.1016/j.pedneo.2011.02.006

[8] Basrur, G.B. (2015) Bilateral Inguinal Hernias Containing Ovaries. Clinics and Practice, 5, Article ID: 708. https://doi.org/10.4081/cp.2015.708

[9] Ozbey, H., Ratschek, M., Schimple, G. and Hollwarth, M.E. (1999) Ovary in Hernia Sac: Prolapsed or a Descended Gonad? Journal of Pediatric Surgery, 34, 977-980. https://doi.org/10.1016/S0022-3468(99)90772-8

[10] Kuera, P. and Glazer, J. (1985) Hydrocele of the Canal of Nuck. A Report of Four Cases. The Journal of Reproductive Medicine, 30, 439-442.

[11] Thomson, G.R. (1984) Complete Congenital Absence of the Vagina Associated with Bilateral Hernia of Uterus, Tubes, and Ovaries. British Journal of Surgery, 36, 99-100. https://doi.org/10.1002/bjs.18003614124

[12] Zitsman, J.L., Cirincione, E. and Margossian, H. (1997) Vaginal Bleeding in an Infant Secondary to Sliding Inguinal Hernia. Obstetrics \& Gynecology, 89, 40-42. https://doi.org/10.1016/S0029-7844(97)00055-0 